\title{
GaAs-based multiple quantum well tunneling injection lasers
}

\author{
X. Zhang, Y. Yuan, A. Gutierrez-Aitken, and P. Bhattacharya \\ Solid State Electronics Laboratory, Department of Electrical Engineering and Computer Science, \\ The University of Michigan, Ann Arbor, Michigan 48109-2122
}

(Received 9 January 1996; accepted for publication 1 August 1996)

\begin{abstract}
We report the modulation characteristics of multiple quantum well tunneling injection lasers designed for $0.98 \mu \mathrm{m}$ emission wavelength. Electrons are injected into the active region through a single barrier via tunneling. The active region has four quantum wells with different well widths. Improved high frequency performance, compared to similar separate confinement heterostructure lasers, has been demonstrated. The modulation response at $21 \mathrm{GHz}$ is above $0 \mathrm{~dB}$ and the extrapolated $-3 \mathrm{~dB}$ modulation bandwidth is $\sim 30 \mathrm{GHz}$ under pulsed bias. (C) 1996 American Institute of Physics. [S0003-6951(96)00442-1]
\end{abstract}

Carrier transport can significantly affect the high speed performance of quantum well lasers. It has been recognized that the separate-confinement heterostructure (SCH) imposes inherent speed limitations on quantum well lasers due to the dynamics of carrier transport mechanisms such as thermalization of carriers injected from the cladding layers, drift and diffusion of carriers to the quantum wells and capture of carriers from a three-dimensional (3D) region in the barriers into a two-dimensional (2D) quantum well. ${ }^{1-3}$ If the electron-hole recombination time by stimulated emission for a laser at high injection approaches the relaxation time, the carrier distribution in the quantum well can become "hot" and is not described by a quasi-Fermi distribution, ${ }^{4}$ causing hot-carrier related problems such as gain compression, enhanced Auger rates etc.

Recently, we demonstrated a novel single quantum well (SQW) tunneling injection laser to overcome the carrierrelaxation induced limitations of $\mathrm{SCH}$ lasers. ${ }^{4,5}$ Carriers are injected from an injection layer through a double barrier tunneling well into the active lasing well, where stimulated emission occurs. The carrier distribution in the lasing well is governed by the thermalization time of carriers injected by tunneling $(\sim 2 \mathrm{ps}){ }^{6}$ rather than by the relaxation time of carriers injected over the barriers, leading to a "cold" carrier distribution even at high injection levels. Differential gain as high as $5.5 \times 10^{-16} \mathrm{~cm}^{2}$ was measured and a modulation bandwidth of $12.5 \mathrm{GHz}$ for a SQW device was achieved in an $\mathrm{In}_{0.2} \mathrm{Ga}_{0.8} \mathrm{As} / \mathrm{GaAs} / \mathrm{AlGaAs} 0.98 \mu \mathrm{m}$ tunneling injection laser. ${ }^{5}$ It is known that the modulation bandwidth of quantum well lasers is larger than the bandwidth of doubleheterostructure (DH) lasers. This is essentially attributed to a large differential gain. In quantum well structures the differential gain decreases as the carrier density increases. For a given value of modal gain, the differential gain increases as the number of quantum wells increases because the carrier density associated with each quantum well decreases as the number of quantum wells increases. Improved high frequency performance is achieved in MQW lasers due to the differential gain enhancement. ${ }^{7}$

In this letter, we report the characteristics of MQW tunneling injection lasers. Such a device is essential for achieving enhanced performance in terms of differential gain and modulation bandwidth. It would also enable the extension of the tunneling concept to a MQW laser. We have character- ized a GaAs-based MQW tunneling injection laser and measured a modulation response greater than $0 \mathrm{~dB}$ at $21 \mathrm{GHz}$. The measured modulation bandwidth of the device is significantly larger than that of a SQW tunneling injection laser and is also larger than the bandwidth of a comparable $\mathrm{SCH}$ laser.

Figure 1(a) shows the schematic cross section of the GaAs-based MQW tunneling injection laser structure. The device structure consists of a $0.5-\mu \mathrm{m}$-thick $n^{+}$-GaAs buffer, a $1-\mu \mathrm{m}$-thick $n-\mathrm{Al}_{x} \mathrm{Ga}_{1-x} \mathrm{As}\left(x=0.4\right.$ or $0.6, n=5 \times 10^{17}$ $\mathrm{cm}^{-3}$ ) outer cladding layer, a $0.1-\mu \mathrm{m}$-thick undoped GaAs $n$-side inner cladding or electron injection layer, a $30 \AA$ undoped single AlAs tunneling barrier, the active region consisting of four undoped $\operatorname{In}_{0.2} \mathrm{Ga}_{0.8}$ As quantum wells with different well widths $(64,54,50$, and $48 \AA$ with $70 \AA$ GaAs barriers in between), a $0.1-\mu$ m-thick undoped $\mathrm{Al}_{0.1} \mathrm{Ga}_{0.9} \mathrm{As}$ $p$-side inner cladding layer, a 1- $\mu$ m-thick $p$ - $\mathrm{Al}_{x} \mathrm{Ga}_{1-x} \mathrm{As}$ $\left(x=0.4\right.$ or $\left.0.6, p=5 \times 10^{17} \mathrm{~cm}^{-3}\right)$ outer cladding layer and finally a $0.2-\mu \mathrm{m}$-thick $p^{+}-\mathrm{GaAs}\left(3 \times 10^{19} \mathrm{~cm}^{-3}\right)$ top contact layer. The structure was grown on (100) semi-insulating GaAs substrate in a RIBER MBE32 system. The cladding layers were grown at a substrate temperature of $680^{\circ} \mathrm{C}$. The GaAs and InGaAs quantum wells were grown at $540{ }^{\circ} \mathrm{C}$. $\mathrm{AlGaAs}$ layers with different $\mathrm{Al}$ compositions were grown by using two effusion cells at different temperatures. No growth interruption was used for adjusting temperatures for the cells.

Figure 1(b) shows the energy band diagram of the MQW tunneling injection laser structure under an applied forward bias, which was obtained by solving the time-independent Schrodinger equation. The dashed lines are the wave functions of the first five states. It can be seen that the wave functions of the first four states are localized in the four quantum wells and the wave function of the fifth state, which is the ground state in the cladding layer, is localized in the injection layer (inner cladding layer on the $n$-side). The widths of the quantum wells and the thickness of the GaAs barrier are optimized so that each of the four wave functions in the quantum wells is not localized in an individual well but distributed in the MQW and the energies of these four states are very close to each other. The maximum energy difference between them is only $11 \mathrm{meV}$. Carriers injected into the active region by optical phonon assisted tunneling are uniformly distributed in the MQW, resulting in a high 


\begin{tabular}{|c|c|c|}
\hline $2000 \AA$ & $\mathrm{p}^{+}-$GaAs & $3 \times 10^{19} \mathrm{~cm}^{-3}$ \\
\hline $1 \mu \mathrm{m}$ & $p-A l_{0.6} \mathrm{Ga}_{0.4} \mathrm{As}$ & $5 \times 10^{17} \mathrm{~cm}^{-3}$ \\
\hline $1000 \dot{A}$ & $\mathrm{i}-\mathrm{Al}_{0.1} \mathrm{Ga}_{0.9} \mathrm{As}$ & undoped \\
\hline $48 \dot{A}$ & $\mathrm{i}-\ln _{0.2} \mathrm{Ga}_{0.8} \mathrm{As}$ & undoped \\
\hline $70 \dot{A}$ & i - GaAs & undoped \\
\hline $50 \AA$ & $1-\ln _{0.2} \mathrm{Ga}_{0.8} \mathrm{As}$ & undoped \\
\hline $70 \AA$ & i-GaAs & undoped \\
\hline $54 \dot{A}$ & $\mathrm{i}-\ln _{0.2} \mathrm{Ga}_{0.8} \mathrm{As}$ & undoped \\
\hline $70 \AA$ & $\mathrm{i}$ - GaAs & undoped \\
\hline $64 \dot{A}$ & $i-\ln _{0.2} \mathrm{Ga}_{0.8} \mathrm{As}$ & undoped \\
\hline $30 \dot{A}$ & i - AlAs & undoped \\
\hline $1000 \AA$ & i - GaAs & undoped \\
\hline $1 \mu \mathrm{m}$ & $\mathrm{n}-\mathrm{Al}_{0.6} \mathrm{Ga}_{0.4} \mathrm{As}$ & $5 \times 10^{17} \mathrm{~cm}^{-3}$ \\
\hline $5000 \dot{A}$ & $n^{+}-$GaAs & $5 \times 10^{18} \mathrm{~cm}^{-3}$ \\
\hline
\end{tabular}

SI - GaAs Substrate

(a)

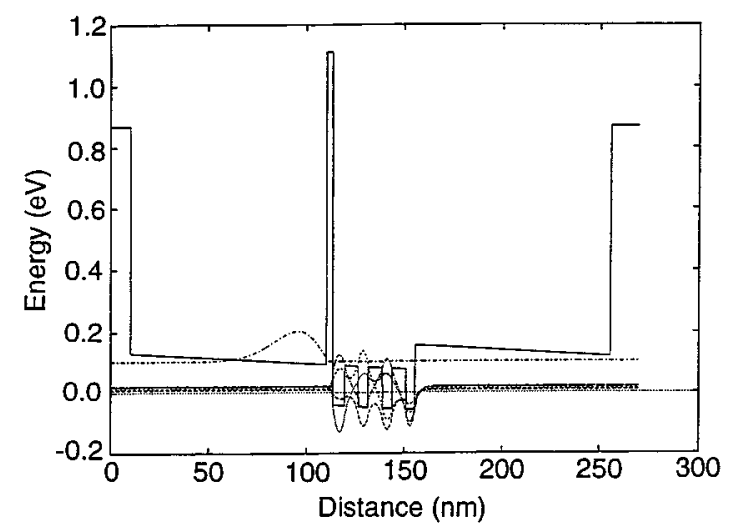

(b)

FIG. 1. (a) Heterostructure grown by molecular beam epitaxy, and (b) conduction band diagram and electron wave functions of the GaAs/ $\mathrm{In}_{0.2} \mathrm{Ga}_{0.8} \mathrm{As} / \mathrm{AlGaAs} \mathrm{MQW}$ tunneling injection laser.

differential gain. The calculated tunneling energy is about 72 meV above the lasing subbands in the MQW. The exact mechanism of transport is under investigation using timeresolved femtosecond differential transmission spectroscopy.

Ridge waveguide lasers were fabricated with a $3 \mu \mathrm{m}$ wide stripe in a coplanar ground-signal-ground contact geometry, ${ }^{8}$ suitable for on-wafer microwave measurements.

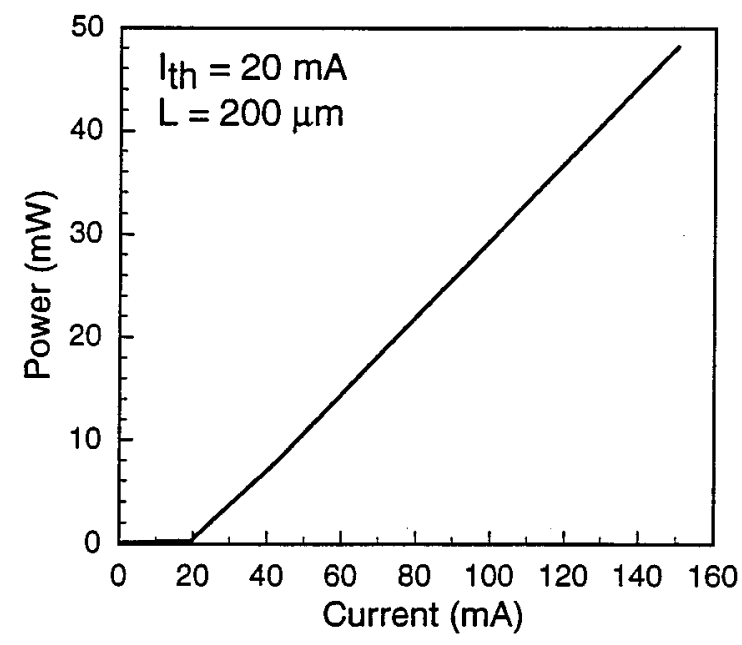

FIG. 2. Light-current characteristics of the $\mathrm{GaAs} / \mathrm{In}_{0.2} \mathrm{Ga}_{0.8} \mathrm{As} / \mathrm{AlGaAs}$ MQW tunneling injection laser under pulsed bias.

The lasers were cleaved to a length of $200 \mu \mathrm{m}$ and mounted onto copper heatsinks. The lasers were pulse biased with $1 \mu$ s pulses having a $1 \%$ duty cycle. The measurement was made at heatsink temperatures of $25^{\circ} \mathrm{C}$. The light-current characteristics of the MQW tunneling injection laser is shown in Fig. 2. The typical threshold current is $20 \mathrm{~mA}$. This value is slightly high, which we believe is due to the nonoptimal quality of the AlGaAs cladding layers. The slope efficiency is $0.34 \mathrm{~mW} / \mathrm{mA}$ per uncoated facet. The peak of the laser emission is at $\sim 0.98 \mu \mathrm{m}$, which confirms lasing from the MQW region. Figure 3 shows the modulation frequency response of the MQW tunneling injection laser. The $-3 \mathrm{~dB}$ modulation bandwidth increases with the increase of the drive current. The maximum modulation bandwidth was achieved at a drive current of $190 \mathrm{~mA}$. The measurement was made up to $21 \mathrm{GHz}$, which is limited by the amplifier and the modulation response is still above $0 \mathrm{~dB}$. The resonance frequency $f_{r}$ at the highest drive current is $12 \mathrm{GHz}$. the extrapolated $-3 \mathrm{~dB}$ modulation bandwidth is $\sim 30 \mathrm{GHz}$, which is much larger than the best modulation bandwidth of $12.5 \mathrm{GHz}$ obtained from a SQW tunneling injection laser. ${ }^{5}$ For the purpose of comparison, we also fabricated GaAs/

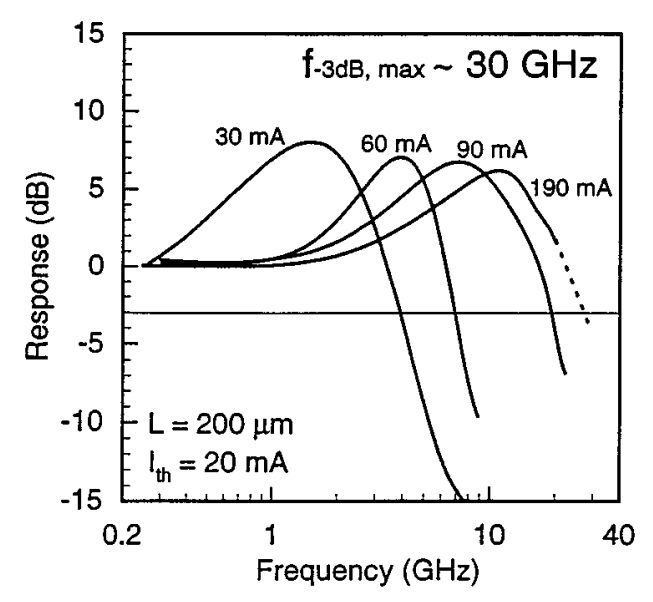

FIG. 3. Small-signal modulation frequency response of the GaAs/ $\mathrm{In}_{0.2} \mathrm{Ga}_{0.8} \mathrm{As} / \mathrm{AlGaAs} \mathrm{MQW}$ tunneling injection laser under pulsed bias. 
$\mathrm{In}_{0.2} \mathrm{Ga}_{0.8} \mathrm{As}$ MQW SCH lasers with four $50 \AA$ wells. There is no tunneling barrier in the structure and the $p$-side inner cladding layer is $0.1 \mu \mathrm{m}$-thick undoped GaAs. For a 4-quantum well SCH laser, a uniform well width provides the best carrier distribution. The other layers are the same as in Fig. 1(a). The maximum modulation bandwidth of this $\mathrm{SCH}$ laser is $16 \mathrm{GHz}$. It is therefore evident that enhanced modulation performance has been achieved from the MQW tunneling injection laser.

The pursuit of better performance, in terms of modulation frequency, in semiconductor lasers has invoked many techniques in the recent past. Higher differential gain can be obtained by increasing the number of quantum wells. Thin inner cladding layers are employed in the laser structure to reduce the influence of carrier transport across the $\mathrm{SCH}$ region on modulation performance ${ }^{9}$ and a modulation bandwidth of $26 \mathrm{GHz}$ was reported from InP based lasers. ${ }^{10}$ Modulation doped MQW lasers are designed to enhance the differential gain by controlling the quasi-Fermi levels in the band structure. ${ }^{11}$ A modulation bandwidth of $37 \mathrm{GHz}$ was reported from $p$-type modulation GaAs/InGaAs MQW lasers. ${ }^{12} \mathrm{We}$ believe that the incorporation of these features into the tunneling MQW laser structure will allow the achievement of modulation bandwidth higher than $40 \mathrm{GHz}$. This work is in progress.

In conclusion, we have demonstrated the modulation characteristics of a MQW tunneling injection laser, where electrons are injected into the active region by tunneling through a thin AlAs barrier. The carriers are uniformly distributed in the multiple quantum wells and improved high frequency performance has been achieved, compared to a similar $\mathrm{SCH}$ structure.

The authors acknowledge Dr. M. Stroscio and Professor J. Singh for useful discussions. The help provided by Dr. J. P. Sun is also acknowledged. This work is supported by the Ballistic Missiles Development Organization, the Advanced Research Project Agency, the Army Research Office, and the Office of Naval Research.

${ }^{1}$ S. Morin, B. Deveaud, F. Clerot, K. Fujiwara, and K. Mitsunaga, IEEE J. Quantum Electron. 27, 1969 (1991).

${ }^{2}$ C. Kan, D. Vassilovski, T. C. Wu, and K. Y. Lau, Appl. Phys. Lett. 61, 752 (1992).

${ }^{3}$ R. Nagarajan, M. Ishikawa, T. Fukushima, R. S. Geels, and J. E. Bowers, IEEE J. Quantum Electron. 28, 1990 (1992).

${ }^{4}$ H. C. Sun, L. Davis, S. Sethi, J. Singh, and P. Bhattacharya, IEEE Photonics Technol. Lett. 5, 870 (1993).

${ }^{5}$ L. Davis, H. C. Sun, H. Yoon, and P. Bhattacharya, Appl. Phys. Lett. 64, 3222 (1994).

${ }^{6}$ C. Y. Sung, T. B. Norris, X. Zhang, Y. L. Lam, I. Vurgaftman, J. Singh, and P. Bhattacharya, presented at the Modulated Semiconductor Structures Conference, Spain, July 1995 (to be published).

${ }^{7}$ B. Zhao, T. R. Chen, and A. Yariv, IEEE Photonics Technol. Lett. 4, 124 (1992)

${ }^{8}$ S. D. Offsey, W. J. Schaff, P. J. Tasker, and L. F. Eastman, IEEE Photonics Technol. Lett. 2, 9 (1990).

${ }^{9}$ R. Nagarajan, T. Fukushima, M. Ishikawa, J. E. Bowers, R. S. Geels, and L. A. Coldren, IEEE Photonics Technol. Lett. 4, 121 (1992).

${ }^{10}$ H. Hillmer, A. Greiner, F. Steinhagen, R. Losch, W. Schlapp, E. Binder, T. Kuhn, and H. Burkhard, presented at the 9th International Conference on Hot Carriers in Semiconductors, Chicago, July 1995 (to be published).

${ }^{11}$ K. Uomi, J. Appl. Phys. 29, 81 (1990).

${ }^{12}$ S. Weisser, E. C. Larkins, K. Czotscher, W. Benz, J. Daleiden, J. Fleissner, M. Maier, J. D. Ralston, B. Romero, A. Schonfelder, and J. Rosenzweig, Proceedings of LEOS 1995, Vol. 2, p. 91. 